LAPTH-1033/04, IFIC/04-11, PCC-0413

\title{
Probing neutrino masses with future galaxy redshift surveys
}

\author{
Julien Lesgourgues \\ Laboratoire de Physique Théorique LAPTH (CNRS-Université de Savoie), \\ B.P. 110, F-74941 Annecy-le-Vieux Cedex, France \\ Sergio Pastor \\ Instituto de Física Corpuscular (CSIC-Universitat de València), \\ Ed. Institutos de Investigación, Apdo. 22085, E-46071 Valencia, Spain \\ Laurence Perotto \\ Physique Corpusculaire et Cosmologie (CNRS-IN2P3), \\ 11 place Marcelin Berthelot, 75231 Paris Cedex 05, France
}

(Dated: October 8, 2018)

\begin{abstract}
We perform a new study of future sensitivities of galaxy redshift surveys to the free-streaming effect caused by neutrino masses, adding the information on cosmological parameters from measurements of primary anisotropies of the cosmic microwave background (CMB). Our reference cosmological scenario has nine parameters and three different neutrino masses, with a hierarchy imposed by oscillation experiments. Within the present decade, the combination of the Sloan Digital Sky Survey (SDSS) and CMB data from the PLANCK experiment will have a $2 \sigma$ detection threshold on the total neutrino mass close to $0.2 \mathrm{eV}$. This estimate is robust against the inclusion of extra free parameters in the reference cosmological model. On a longer term, the next generation of experiments may reach values of order $\sum m_{\nu}=0.1 \mathrm{eV}$ at $2 \sigma$, or better if a galaxy redshift survey significantly larger than SDSS is completed. We also discuss how the small changes on the free-streaming scales in the normal and inverted hierarchy schemes are translated into the expected errors from future cosmological data.
\end{abstract}

PACS numbers: 14.60.Pq, 95.35.+d, 98.80.Es

\section{INTRODUCTION}

Neutrino physics has provided the first clear indication of particle physics beyond the Standard Model, since we have experimental evidences for non-zero neutrino masses. Analyses of data from atmospheric and solar neutrino experiments have shown the allowed regions for the squared mass differences $\left(\Delta m_{\nu}^{2}\right)$ at two different scales. Such values will be known with better precision in the next years, in particular for the larger atmospheric $\Delta m_{\nu}^{2}$ using the results of future long-baseline oscillation experiments.

However, from oscillation experiments no information can be obtained on the absolute values of neutrino masses, since the lightest neutrino mass remains unconstrained. Tritium decay experiments tell us that each neutrino mass cannot be larger than $2.2 \mathrm{eV}(95 \% \mathrm{CL})$ at present [1], to be improved to $\sim 0.35 \mathrm{eV}$ with KATRIN [2]. More stringent bounds exist from experiments searching for neutrinoless double beta decay, that will be improved in the near future [3], but unfortunately they depend on the details of the neutrino mixing matrix.

Cosmology offers several advantages: the cosmic neutrino background provides an abundant density of relic neutrinos with an equal momentum distribution for all flavors (up to $1 \%$ corrections), which implies that mixing angles have no effect. Although neutrinos cannot be the dominant dark matter component, they can still constitute a small, hot part of the matter density producing an erasure of perturbations at small scales through their free-streaming effect (for a review, see e.g. [4]). A comparison with data from the large scale structure (LSS) of the Universe is thus sensitive to neutrino masses, as emphasized in [5].

At present, cosmological data allow us to bound the total neutrino mass to values of $\sum m_{\nu} \lesssim 0.6-1.0 \mathrm{eV}$ 6, 7, 8, 9, 10, 11, 12], depending on the data and priors used. These ranges already compromise the 4 neutrino scenarios that could explain the additional large neutrino mass difference required by the LSND results (that also imply a fourth, sterile neutrino), but is not yet capable of reaching the necessary $0.1 \mathrm{eV}$ range in order to test the hierarchical 3 neutrino schemes. But such small masses could be detected in the next future when more precise cosmological data are available, in a parallel effort to those of beta and double beta decay experiments on Earth.

In this paper we analyze the future sensitivities of cosmological data to neutrino masses, extending the pioneering work [5] and in particular the detailed analysis in [13] (see also [14]), that was more recently updated in [15]. In contrast to this last work we consider, in addition to ideal Cosmic Microwave Background (CMB) observations limited only 


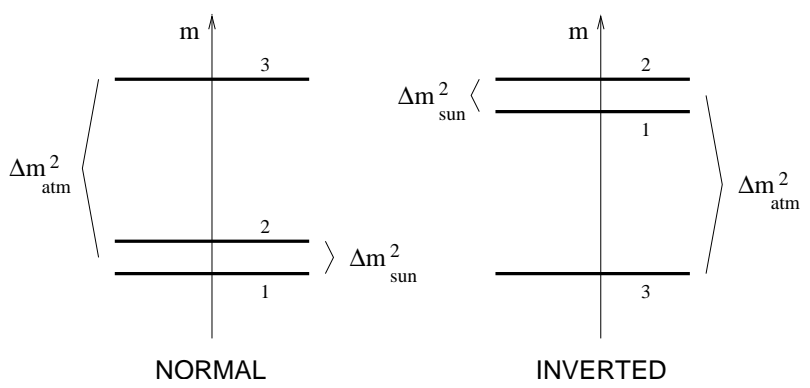

FIG. 1: The two neutrino schemes allowed if $\Delta m_{\mathrm{atm}}^{2} \gg \Delta m_{\mathrm{sun}}^{2}$ : normal hierarchy (NH) and inverted hierarchy (IH).

by cosmic variance, the experimental specifications of satellite missions such as PLANCK and the mission concept CMBpol (Inflation Probe), as well as ground-based detectors such as ACT or SPTpol, that will extend the PLANCK data to smaller angular scales. We also increase the number of cosmological parameters of previous analyses, including also the helium fraction, extra relativistic degrees of freedom, spatial curvature, dark energy with constant equation of state, or a primordial spectrum with running tilt. Finally, our work is the first one in which it is assumed that neutrinos have three different masses, in order to compute accurately the free-streaming effect associated to the mass schemes allowed by oscillation experiments.

Note that throughout this work, we will assume that the LSS power spectrum is measured solely with galaxy redshift surveys. For complementary constraints based on gravitational lensing, we refer the reader to Refs. 16, 17].

This paper is organized as follows. In Sec. II we review the expected values of neutrino masses and their impact on Cosmology. We describe future CMB experiments and galaxy surveys in Sec. III and the method to forecast the errors on cosmological parameters in Sec. IV. Finally, we present our results in Sec. V, with a summary and conclusions in Sec. VI.

\section{NEUTRINO MASSES}

Nowadays we have experimental evidences for neutrino oscillations from solar and atmospheric neutrino detectors, recently also supported from data on neutrinos from artificial sources (Kamland and K2K). Detailed analyses of the experimental data lead to the following values of the mass squared differences (best fit values $\pm 3 \sigma$ ranges)

$$
\begin{aligned}
& \Delta m_{\mathrm{atm}}^{2}=\Delta m_{32}^{2}=\left(2.6_{-1.2}^{+1.1}\right) \times 10^{-3} \mathrm{eV}^{2} \\
& \Delta m_{\mathrm{sun}}^{2}=\Delta m_{21}^{2}=\left(6.9_{-1.5}^{+2.6}\right) \times 10^{-5} \mathrm{eV}^{2}
\end{aligned}
$$

taken from [18]. These ranges are only slightly different in other recent analyses, see e.g. [19, 20], while a lower $\Delta m_{\mathrm{atm}}^{2}$ seems required by new Super-Kamiokande data and 3-dimensional atmospheric fluxes. The errors in the above equation will be significantly reduced with new data from Kamland in the case of $\Delta m_{21}^{2}$, and with data from future long-baseline oscillation experiments such as MINOS, ICARUS and OPERA, which will give the atmospheric $\Delta m^{2}$ with $10 \%$ accuracy (reduced to $5 \%$ with the superbeam proposal JPARC-SK) [21]. Current data also provide the allowed ranges of the neutrino mixing angles $\theta_{12}$ and $\theta_{23}$, and an upper bound on $\theta_{13}$.

Indications for a third, heavier $\Delta m_{\nu}^{2}$ exist from the LSND experiment [22], implying a fourth (sterile) neutrino. Such a mass is already being tested by present cosmological data, although not ruled out yet [7, 8, 11, 12], and the LSND results will be checked by the ongoing experiment MiniBoone. Here we choose not to include such a large $\Delta m_{\nu}^{2}$ and consider only the values in Eq. [1

The three neutrino masses that lead to the values in Eq. 1 1 can be accommodated in two different neutrino schemes,

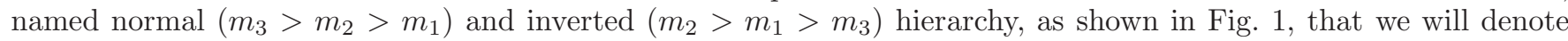
$\mathrm{NH}$ and IH. At present we have no indication of which scheme is the correct one. However, it has been suggested that some information could be extracted from future data from Supernova neutrinos, very large baseline oscillation experiments, or neutrinoless double beta decay searches if the effective $m_{\nu}$ is below some threshold (for reviews, see e.g. [23, 24]). In general, determining the type of mass spectrum depends on the precision with which the other mixing parameters would be measured.

Relic neutrinos were created in the Early Universe and decoupled from the rest of the plasma when the temperature dropped below $\sim 1 \mathrm{MeV}$, when they were ultra-relativistic. After decoupling all neutrino flavors kept a Fermi-Dirac spectrum, only distorted at percent level during the process of electron-positron annihilations into photons [25, 26]. It is well-known that massive neutrinos could account for a significant fraction of the total energy density of the 


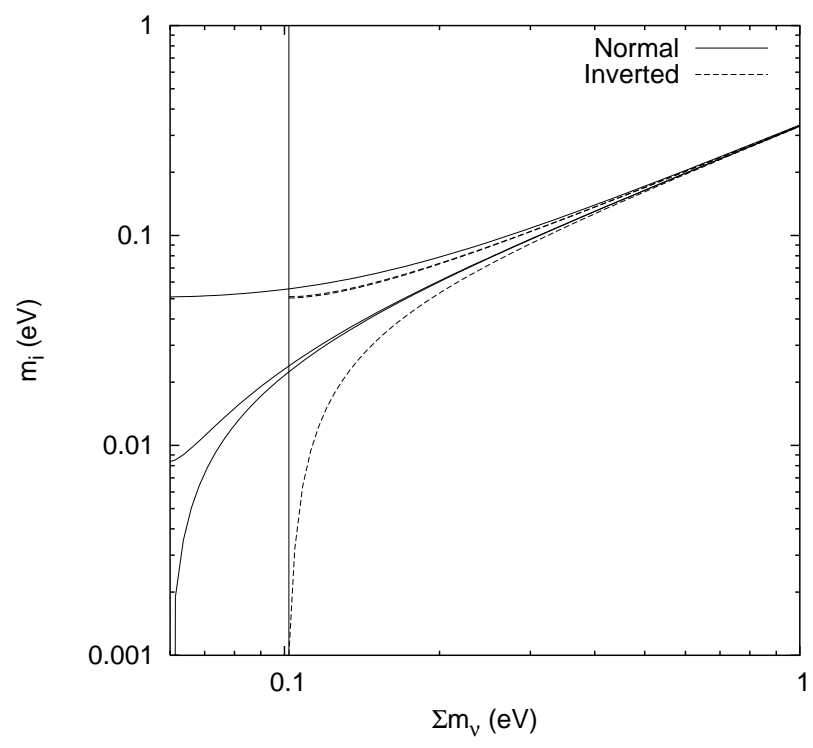

FIG. 2: Neutrino masses as a function of the total mass in the two schemes for the best-fit values of $\Delta m^{2}$ in Eq. 1 The vertical line marks the smallest value of $\sum m_{\nu}$ in the inverted scenario.

Universe today, being their contribution directly proportional to the number density. For vanishing neutrino chemical potentials, the total neutrino contribution to the critical density is given by

$$
\Omega_{\nu}=\frac{\sum m_{\nu}}{93.2 \mathrm{eV}} h^{-2}
$$

where $h$ is the Hubble constant in units of $100 \mathrm{~km} \mathrm{~s}^{-1} \mathrm{Mpc}^{-1}$ and $\sum m_{\nu}$ runs over all neutrino mass states. For fixed neutrino masses, $\Omega_{\nu}$ would be enhanced if neutrinos decoupled with a significant chemical potential (or equivalently, for large relic neutrino asymmetries), but this possibility is now ruled out [27].

Therefore cosmology is at first order sensitive to the total neutrino mass $\sum m_{\nu}=m_{1}+m_{2}+m_{3}$ (for the 3 neutrino schemes that we consider), but blind to the neutrino mixing angles or possible CP violating phases. This fact differentiates cosmology from terrestrial experiments such as beta decay and neutrinoless double beta decay, which are sensitive to $\sum_{i}\left|U_{e i}\right|^{2} m_{i}^{2}$ and $\left|\sum_{i} U_{e i}^{2} m_{i}\right|$, respectively, where $U$ is the $3 \times 3$ mixing matrix that relates the weak and mass bases.

It is interesting to see how the total mass is distributed among the neutrino states for the two different schemes described above. They are plotted in Fig. 2] For a total mass above $\sim 0.2-0.3 \mathrm{eV}$ the two schemes are similar and correspond to a degenerate scenario where each mass is $\sum m_{\nu} / 3$. However, for smaller masses the number of neutrino states with relevant masses is 2 (1) in the inverted (normal) hierarchy.

The effect of neutrino masses on cosmological observables has been usually considered equivalent for fixed $\sum m_{\nu}$ (or $\Omega_{\nu} h^{2}$ ). However, many papers noted in the past that this is not the case and could potentially lead to differences, i.e. the neutrino mass spectrum should be incorporated if the sensitivity to neutrino masses is good enough (see, for instance the comments in 15, 16, 28]). As an example, we note that in the mid-1990s it was shown that for CHDM models with the same total neutrino mass (of order some eVs), those with two degenerate massive neutrinos fitted better the data than those with only one (see e.g. [29]).

Fixed the total neutrino mass, a different distribution among the 3 states $\left(m_{1}, m_{2}, m_{3}\right)$ causes a slight modification of the transit from a relativistic to a non-relativistic behavior. This can be seen in Fig. 3 where the evolution of the neutrino energy density is plotted for several cases with the same total neutrino mass, equally shared by 1,2 or 3 neutrino states, as well as the realistic NH and IH schemes (taking the best-fit values of $\Delta m^{2}$ ). Therefore, the evolution of background quantities is not completely independent of the mass splitting. However, the main difference appears at the level of perturbations. Indeed, in the case of non-degenerate massive neutrinos, various free-streaming scales are imprinted in the matter power spectrum $P(k)$. This is illustrated in Fig. 团 where we compare $P(k)$ in the same cases as in Fig. [3] These results were obtained with our modified version of the public code CMBFAST [30] (see section $\nabla$ for details).

We have recently summarized the effects of massive neutrinos on cosmological observables in [12]. Here we simply remind that only neutrinos with masses close to the recombination temperature $\left(T_{\mathrm{dec}} \sim 0.3 \mathrm{eV}\right)$ leave an imprint on 


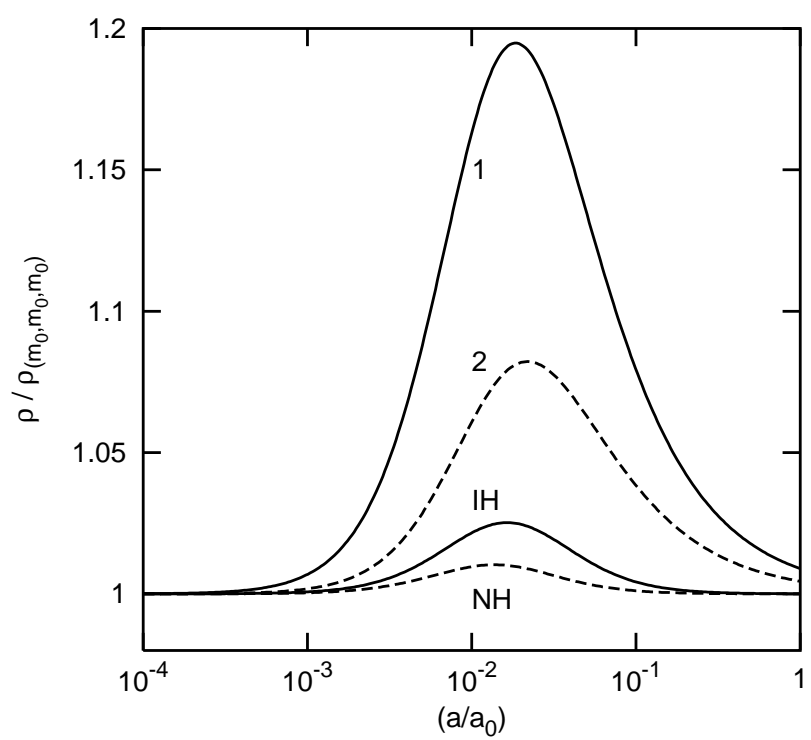

FIG. 3: Evolution of the total neutrino energy density as a function of the scale factor of the Universe for models where the same $\sum m_{\nu}(0.12 \mathrm{eV})$ is distributed differently. Each line corresponds to the energy density of 4 different cases (only 1 or 2 massive states, Normal and Inverted Hierarchy) normalized to the case with 3 massive states with mass $m_{0}=\sum m_{\nu} / 3$.

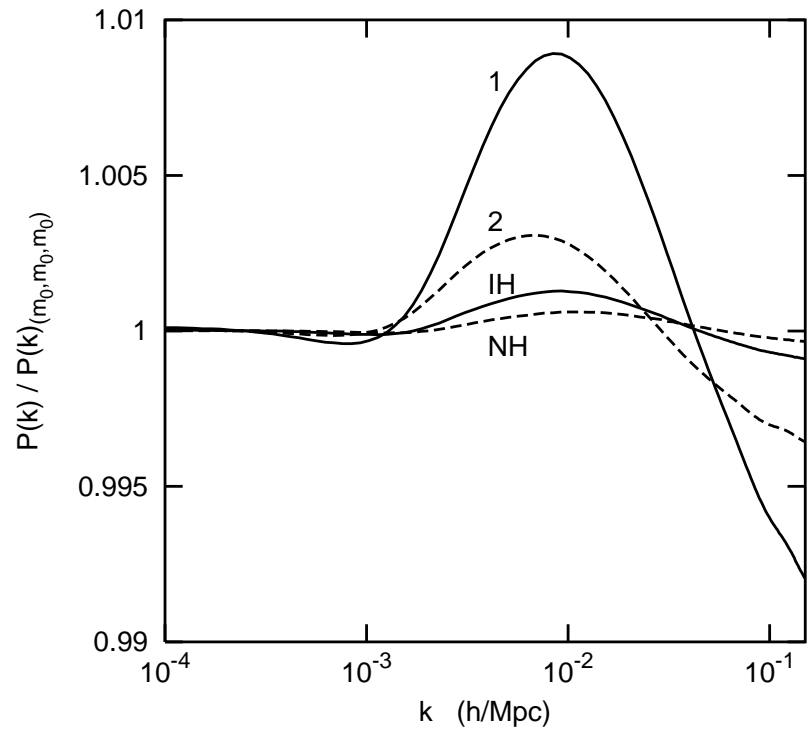

FIG. 4: Comparison of the matter power spectrum obtained for various models where the same $\sum m_{\nu}(0.12 \mathrm{eV})$ is distributed differently. The four lines correspond to the cases with 1 or 2 massive states, Normal and Inverted Hierarchy, divided each time by that with 3 massive states of equal mass $m_{0}=\sum m_{\nu} / 3$. Differences in the various individual masses and free-streaming scales affect the position and amplitude of the break in the power spectrum.

the CMB angular spectra, while neutrinos with smaller masses have almost the same effect as massless neutrinos. On the other hand, the dominant effect is the one induced by free-streaming on the matter power spectrum. Therefore, the usual strategy is to combine CMB and LSS measurements, where the former roughly fix most of the cosmological parameters, while the latter is more sensitive to neutrino masses.

\section{FUTURE CMB AND LSS DATA}

In this section we briefly describe the experimental projects, planned or in development, that will provide data on the CMB anisotropy spectrum or on the distribution of LSS. 


\section{A. CMB experiments}

The quality of the first-year data from the Wilkinson Microwave Anisotropy Probe (WMAP) 66., complemented by the results of other experiments at smaller angular scales such as ACBAR, CBI or VSA [31, 32, 33], has shown the importance of CMB data as a probe of cosmological parameters. The CMB experiments measure the temperature fluctuations in the sky that can be expanded in spherical harmonics,

$$
\frac{\Delta T}{T}(\theta, \phi)=\sum_{l, m} a_{l m} Y_{l m}(\theta, \phi) .
$$

If the underlying perturbations are Gaussian, all information is encoded in the angular power spectrum $C_{l} \equiv\left\langle\left|a_{l m}\right|^{2}\right\rangle$. In addition the CMB experiments can be sensitive to polarization anisotropies, that are expressed in terms of the angular spectra of the E and B modes of polarization, as well as the temperature polarization cross-correlation (TE) spectrum.

After WMAP, the next satellite mission will be PLANCK ${ }^{1}$, to be launched in 2007, whose experimental parameters are listed in Table凹 After a couple of years, it will provide CMB data more precise than that of WMAP, in particular concerning polarization. We also consider the CMBpol or Inflation Probe mission concept, presented in the framework of NASA's Beyond Einstein Program² ${ }^{2}$ This experiment would have better sensitivity than the limit imposed by cosmic variance (up to $l \sim 2300$ for E-polarization, even beyond for temperature).

In parallel to the satellite missions, there will be ground-based experiments that will measure the CMB at smaller angular scales with significantly smaller sky coverage but good sensitivities, such as SPTpol ${ }^{3}$ (in construction), $\mathrm{ACT}^{4}$ (funded in January 2004), or QUaD [34] (in construction). As an example, we consider SPTpol with the characteristics listed in Table

The observed power spectrum can be decomposed into primary anisotropies, gravitational lensing distortions, and foreground contamination. The central frequencies of CMB detectors are usually chosen in order to minimize the foreground contribution. In addition, by combining various frequencies, future experiments will have the power to separate efficiently the CMB blackbody from the various foregrounds contributions, even on small angular scales where the latter start to be significant. It is possible to build models for the foregrounds and to predict their impact on parameter extraction [34, 35, 36]; this approach is rather model-dependent, since the level of many foreground signals has not yet been measured experimentally. Here, we will not enter into such details. When dealing with PLANCK, we will employ only three frequency channels from the high frequency instrument (HFI), making the (usual) simplifying assumption that other channels will be used for measuring the various foregrounds, and for cleaning accurately the primary signal. We will do similar assumptions for SPTpol and CMBpol. We will also speculate on the results of an "ideal CMB experiment" limited only by cosmic variance. Then, we will limit ourselves to $l_{\max }=2500$ both for temperature and polarization, which assumes an efficient method for foreground subtraction - in particular of point-like sources and dust - but remains realistic (as indicated by Fig. 7 in [35]). For the two satellite experiments, we assume a sky coverage of $f_{\text {sky }}=0.65$, which represents a conservative estimate of the data fraction that will be included in the analysis in order to avoid galactic foregrounds. For the "ideal CMB experiment", we adopt the more optimistic value $f_{\text {sky }}=1$, assuming that all galactic foregrounds can be subtracted (see e.g. the component separation method described in [37]).

The issue of gravitational lensing distortion is subtle and potentially very interesting. Since lensing is induced by large scale structure, mainly on linear scales, this effect can be accurately predicted for a given matter power spectrum. Therefore, if the gravitational distortion of the CMB maps could be measured directly, there would be an opportunity to extract the matter power spectrum (and the underlying cosmological parameters) independently from redshift surveys. A way of doing this is described in [38, 39, 40], and has been already applied to future neutrino mass extraction by [17]. Here, we do not incorporate this method, and assume that the matter power spectrum is measured only with redshift surveys, leaving a combined analysis for the future. Therefore, throughout the analysis, we will employ the unlensed CMB power spectra ${ }^{5}$. For the T, E and TE modes, lensing distortions are subdominant. In contrast, for the B mode, lensing is expected to dominate over the primary anisotropies at least on small angular scales. The angle above which lensing is subdominant crucially depends on the tensor-to-scalar ratio, an inflationary

\footnotetext{
1 http://www.rssd.esa.int/index.php?project=PLANCK

2 http://universe.gsfc.nasa.gov/program/inflation.html

3 http://astro.uchicago.edu/spt/

4 http://www.hep.upenn.edu/ angelica/act/act.html

5 Note that including lensing corrections is technically easy with CMBFAST. However, this would introduce some correlations among different modes and scales that would artificially lower the predicted errors on each cosmological parameters [17, 38].
} 


\begin{tabular}{|c|c|c|c|c|c|}
\hline Experiment & $f_{\text {sky }}$ & $\nu$ & $\theta_{b}$ & $\Delta_{T}$ & $\overline{\Delta_{P}}$ \\
\hline \multirow[t]{3}{*}{ PLANCK } & 0.65 & 100 & $9.5^{\prime}$ & 6.8 & 10.9 \\
\hline & & 143 & $7.1^{\prime}$ & 6.0 & 11.4 \\
\hline & & 217 & $5.0^{\prime}$ & 13.1 & 26.7 \\
\hline SPTpol & 0.1 & 217 & $0.9^{\prime}$ & 12 & 17 \\
\hline CMBpol & 0.65 & 217 & $3.0^{\prime}$ & 1 & 1.4 \\
\hline
\end{tabular}

TABLE I: Experimental parameters of CMB projects: here $\theta_{b}$ measures the width of the beam, $\Delta_{T, P}$ are the sensitivities per pixel in $\mu \mathrm{K}, \nu$ is the center frequency of the channels in $\mathrm{GHz}$ and $f_{\text {sky }}$ the observed fraction of the sky. For the PLANCK 100 $\mathrm{GHz}$ channel, the value of $\Delta_{P}$ takes into account the recent design with eight polarized bolometers.

parameter which order of magnitude is still unknown. So, we follow a conservative approach and not take the B mode into account. This amounts in assuming that the gravitational wave background generated by inflation is small, so that the B mode gives no information on primary anisotropies.

\section{B. Galaxy surveys}

The existing data on the distribution of galaxies at large scales come from several galaxy surveys, of which the completed $2 \mathrm{dF}$ survey ${ }^{6}$ and the ongoing Sloan Digital Sky Survey ${ }^{7}$ (SDSS) are the largest. SDSS will complete its measurements in 2005. The matter power spectrum $P(k)$ can be reconstructed from the data, which gives an opportunity to test the free-streaming effect of massive neutrinos. However, the linear power spectrum is found modulo a biasing factor $b^{2}$, which reflects the discrepancy between the total matter fluctuations in the Universe, and those actually seen by the instruments. Here we assume that the bias parameter $b$ is independent of the scale $k$.

An important point concerning LSS data is the non-linear clustering of the smallest scales. The usual approach is to discard any information above an effective cut-off wavenumber $k_{\max }$, while considering results at lower $k$ 's as a direct estimate of the linear power spectrum. The cut-off value must be chosen with care: if $k_{\max }$ is too small, we can lose a lot of information, especially concerning the neutrino free-streaming scale. If $k_{\max }$ is too large, we can underestimate the error on cosmological parameters, first by neglecting any theoretical uncertainty in the quasi-linear corrections that could be applied to the spectrum, and second by ignoring the non-gaussianity induced by non-linear evolution 41].

Apart from $k_{\max }$, the important parameter characterizing the galaxy survey is its effective volume in $k$ space, defined in [41]. If the number density of objects in the survey $n(\mathbf{r})$ is roughly constant over the survey volume, and if the observed power spectrum $P(k)$ is bigger than $1 / n$ over the scales of interest (i.e., from the turn-over scale in $P(k)$ up to $\left.k_{\max }\right)$, the effective volume is equal to the actual volume of the survey. This is a reasonable approximation for all the examples that we will consider here. For instance, the SDSS the Bright Red Galaxy (BRG) survey has an effective volume of roughly $V_{\text {eff }} \simeq 1(\mathrm{Gpc} / h)^{3}\left[13\right.$. (which comes from a sky coverage $f_{\text {sky }}=0.25$ and a radial length of $1 \mathrm{Gpc} h^{-1}$ ).

Beyond SDSS, plans for larger surveys are under discussion. For instance, we can mention the Large Synoptic Survey Telescope $^{8}$ (LSST), which in the future could cover the entire sky and at the same time be capable of measuring fainter objects 42. LSST is designed mainly for weak lensing observations. In order to map the total matter distribution up to half the age of the Universe (i.e., up to a redshift $z \sim 0.8$ or a radial length $l \sim 2.3 \mathrm{Gpc} / h$ ) in a solid angle 30,000 $\operatorname{deg}^{2}\left(f_{\text {sky }} \sim 0.75\right)$, it could measure $2 \times 10^{8}$ redshifts up to $z=1.5$. Inspired roughly by these numbers, at the end of this analysis, we will speculate on the possibility to measure the power spectrum in a effective volume as large as $V_{\text {eff }}=(4 \pi / 3) f_{\text {sky }} l^{3} \sim 40(\mathrm{Gpc} / h)^{3}$.

The mechanism of structure formation affects larger wavelengths at later times. So, in order to measure the linear power spectrum on small scales, it would be very useful to build high redshift galaxy surveys. This is one of the main goals of the Kilo-Aperture Optical Spectrograph (KAOS) proposal ${ }^{9}$. KAOS could build two catalogs centered around redshifts $z=1$ and $z=3$, corresponding roughly to $k_{\max } \sim 0.2 h \mathrm{Mpc}^{-1}$ and $k_{\max } \sim 0.48 h \mathrm{Mpc}^{-1}$ respectively, instead of $k_{\max } \sim 0.1 \mathrm{~h} \mathrm{Mpc}^{-1}$ today (conservative values). In both catalogs, the number density would be such that

\footnotetext{
6 http://www.mso.anu.edu.au/2dFGRS/

7 http://www.sdss.org

8 http://www.lsst.org

9 http://www.noao.edu/kaos
} 
$1 / n \sim P\left(k_{\max }\right)$, and the effective volume of the two samples close to $V_{\text {eff }} \sim 0.5(\mathrm{Gpc} / h)^{3}$ and $V_{\text {eff }} \sim 0.6(\mathrm{Gpc} / h)^{3}$ respectively ${ }^{10}$. This experiment is designed mainly for measuring the scale of baryonic oscillations, in order to constrain dark energy. However, we will see that it would be also appropriate for improving constraints on the neutrino masses.

\section{FORECAST OF FUTURE BOUNDS: FISHER MATRIX ANALYSIS}

Since the characteristics of future CMB experiments and galaxy surveys are already known with some precision, it is possible to assume a "fiducial" model, i.e., a cosmological model that would yield the best fit to the future data, and employ the Fisher matrix method to forecast the error with which each parameter will be extracted. This method has been widely used for many cosmological parameters, some of them related to neutrinos. For instance, we can mention that forecast analyses based on the Fisher matrix have shown that with future data there will be a potential sensitivity to an effective number of neutrinos of the order $\Delta N_{\text {eff }} \sim 0.2$ [43, 44, 45], a value that is complementary to and will eventually improve the accuracy of primordial nucleosynthesis results (see e.g. [46, 47]).

Starting with a set of parameters $x_{i}$ describing the fiducial model, one can compute the angular power spectra of CMB temperature and polarization anisotropies $C_{l}^{X}$, where $X=T, E, T E$. Simultaneously, one can derive the linear power spectrum of matter fluctuations $P(k)$, expanded in Fourier space. The error $\delta x_{i}$ on each parameter can be calculated from the reduced (dimensionless) Fisher matrix $F_{i j}$, which has two terms. The first one accounts for the CMB experiment and is computed according to ref. [48]

$$
F_{i j}(\mathrm{CMB})=\sum_{l=2}^{l_{\max }} \sum_{X, Y} \frac{\partial C_{l}^{X}}{\partial \ln x_{i}} \operatorname{Cov}^{-1}\left(C_{l}^{X}, C_{l}^{Y}\right) \frac{\partial C_{l}^{Y}}{\partial \ln x_{j}},
$$

where $\operatorname{Cov}\left(C_{l}^{X}, C_{l}^{Y}\right)$ is the covariance matrix of the estimators of the corresponding CMB spectrum. For instance, the $T T$ element is given by

$$
\operatorname{Cov}\left(C_{l}^{T}, C_{l}^{T}\right)=\frac{2}{(2 l+1) f_{\mathrm{sky}}}\left[C_{l}^{T}+\left(\sum_{\mathrm{ch} .} \omega_{T} B_{l}^{2}\right)^{-1}\right]^{2}
$$

Here, the first term arises from cosmic variance, while the second is a function of the experimental parameters summed over the channels: $B_{l}^{2}=\exp \left(-l(l+1) \theta_{b}^{2} / 8 \ln 2\right)$ is the beam window function (assumed to be Gaussian), $\theta_{b}$ is the FWHM of the beam and $\omega_{T}=\left(\theta_{b} \Delta_{T}\right)^{-2}$ is the inverse square of the detector noise level $\left(\Delta_{T}\right.$ is the sensitivity per pixel, and the solid angle per pixel can be approximated by $\left.\theta_{b}^{2}\right)$. For the experiments that we consider here, all these numbers can be found in Table \. The other terms of the covariance matrix can be found, for instance, in [13].

The second term of the reduced Fisher matrix accounts for the galaxy survey data and is calculated following Tegmark [1],

$$
F_{i j}(\mathrm{LSS})=2 \pi \int_{0}^{k_{\max }} \frac{\partial \ln P_{\mathrm{obs}}(k)}{\partial \ln x_{i}} \frac{\partial \ln P_{\mathrm{obs}}(k)}{\partial \ln x_{j}} w(k) d \ln k .
$$

Here $w(k)=V_{\text {eff }} /(2 \pi / k)^{3}$ is the weight function of the galaxy survey and we have approximated the lower limit of the integral $k_{\min } \simeq 0$. We defined $P_{\mathrm{obs}}(k) \equiv b^{2} P(k)$, and $k_{\max }$ is the maximal wave number on which linear predictions are reliable. This expression is only an approximation, since in addition to non-linear clustering it ignores edge effects and redshift space distortions.

Inverting the total Fisher matrix, one obtains an estimate of the 1- $\sigma$ error on each parameter, assuming that all other parameters are unknown

$$
\frac{\delta x_{i}}{x_{i}}=\left(F^{-1}\right)_{i i}^{1 / 2}
$$

It is also useful to compute the eigenvectors of the reduced Fisher matrix (i.e., the axes of the likelihood ellipsoid in the space of relative errors). The error on each eigenvector is given by the inverse square root of the corresponding eigenvalue. The eigenvectors with large errors indicate directions of parameter degeneracy; those with the smallest errors are the best constrained combinations of parameters.

10 The characteristics of KAOS are taken from the "Purple Book" available on-line at http://www.noao.edu/kaos 

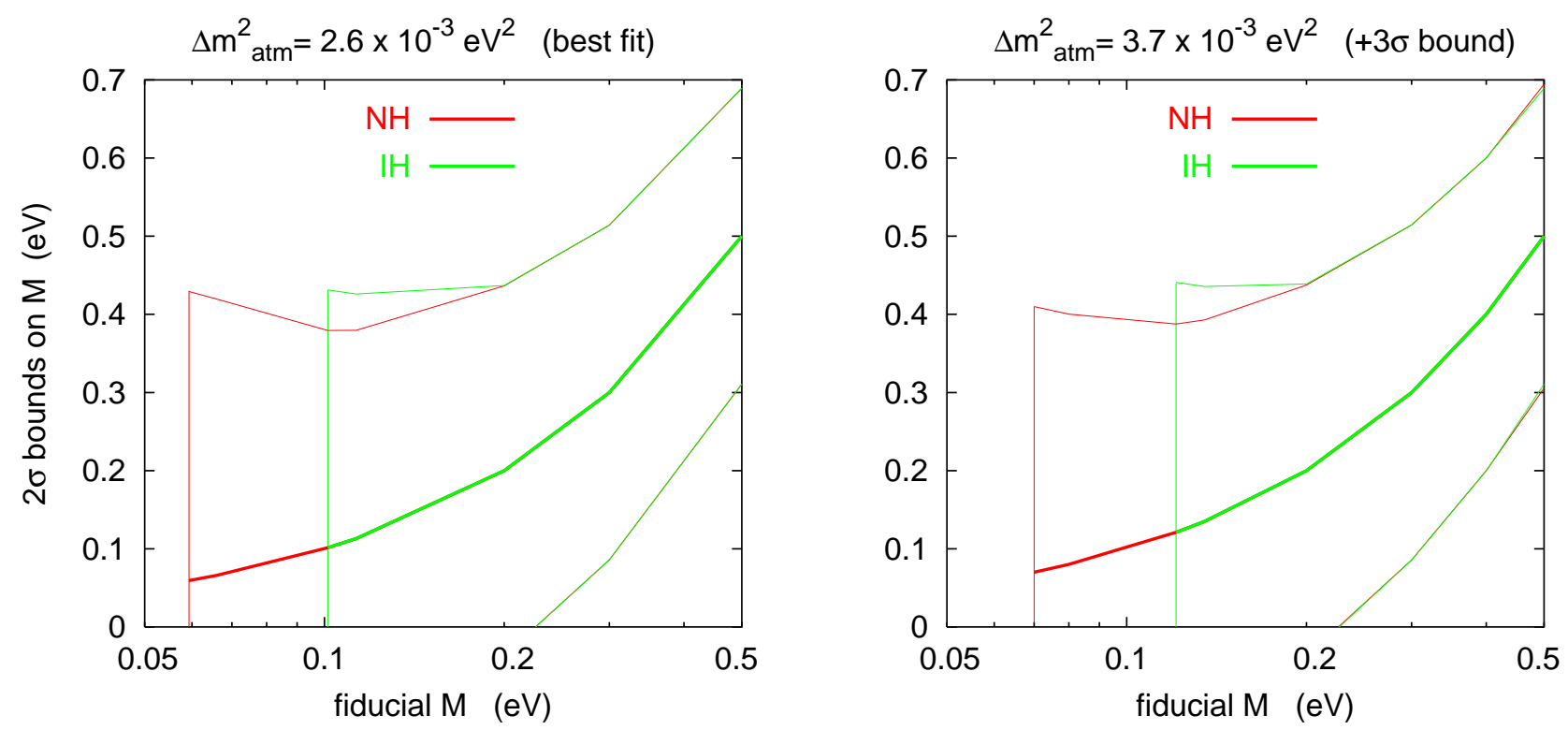

FIG. 5: Predicted $2 \sigma$ error on the total neutrino mass $M \equiv \sum m_{\nu}$ as a function of $M$ in the fiducial model, using PLANCK and SDSS (limited to $k_{\max }=0.15 h \mathrm{Mpc}^{-1}$ ). The left plot was obtained with the preferred experimental value of $\Delta m_{\text {atm }}^{2}$, and the right plot with the current $3 \sigma$ upper bound. In each case, we show the results assuming either $\mathrm{NH}$ or IH.

\section{RESULTS}

We have computed the total Fisher matrix from Eqs. 4and [6] using various experimental specifications. Throughout the analysis, our fiducial model is the concordance "flat $\Lambda \mathrm{CDM}$ " scenario, with parameters close to the current bestfit values and with additional neutrino masses. The nine free parameters with respect to which derivatives are computed are: $\Omega_{m} h^{2}$ (matter density, including baryons, cold dark matter and neutrinos), $\Omega_{b} h^{2}$ (baryon density), $\Omega_{\Lambda}$ (cosmological constant), $C_{200}^{T}$ (amplitude of temperature spectrum at multipole 200), $n_{s}$ (scalar tilt), $\tau$ (optical depth to reionization), $y_{H e}$ (fraction of baryonic mass in the form of Helium), $M \equiv \sum m_{\nu}$ (total neutrino mass) and $b$ (unknown bias of the LSS data). The fiducial value of $b$ is irrelevant by construction, and we will try various values of $M$, distributed following the $\mathrm{NH}$ or $\mathrm{IH}$ scheme. Other fiducial values read:

$$
\left(\Omega_{m} h^{2}, \Omega_{b} h^{2}, \Omega_{\Lambda}, C_{200}^{T}, n_{s}, \tau, y_{H e}\right)=(0.143,0.023,0.70,0.85,0.96,0.11,0.24) .
$$

All derivatives are computed at zero spatial curvature (by varying $h$ appropriately). Note that we use double-sided derivatives with step $10 \%$ for $M, 50 \%$ for $y_{H e}, 5 \%$ for all other parameters. We checked carefully that these steps are sufficient in order to avoid possible numerical errors caused by the limited precision of the Boltzmann code - in our case, version 4.5.1 of CMBFAST [30], with option "high precision". We also checked that with twice larger steps, the results change only by a negligible amount. These conditions were not a priori obvious for the smallest neutrino masses studied here, but we increased the precision of the neutrino sector in CMBFAST accordingly. Actually, in order to study three neutrino species with different masses, we performed significant modifications throughout CMBFAST. For each mass eigenstate, we integrate some independent background and perturbation equations, decomposed in 15 momentum values, up to multipole $l=7$. Finally, we include the small distortions in the neutrino phase-space distributions caused by non-instantaneous decoupling from the electromagnetic plasma (with QED corrections at finite temperature) [26], but these last effects are almost negligible in practice.

\section{A. PLANCK+SDSS}

We first derive the precision with which the combined PLANCK and SDSS data will constrain the total neutrino mass in a near future. Experimental specifications for these experiments are given in the previous section, and we

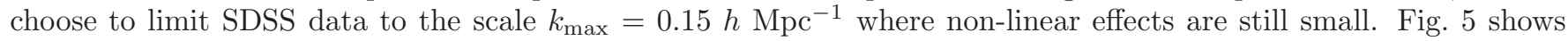
the predicted $2 \sigma$ error on $M$ for various fiducial models, assuming different values of $M$, the two possible schemes for the mass splitting (either $\mathrm{NH}$ or $\mathrm{IH}$ ), and two different values of $\Delta m_{\mathrm{atm}}^{2}$. The solar mass scale $\Delta m_{\text {sun }}^{2}$ is essentially irrelevant in this analysis, and is kept fixed to the current preferred value of $6.9 \times 10^{-5} \mathrm{eV}^{2}$. The possible values of 


\begin{tabular}{lcccccccccc}
\hline \hline & $\ln C_{200}^{T}$ & $n_{s}$ & $\tau$ & $\Omega_{\Lambda}$ & $\Omega_{m} h^{2}$ & $\Omega_{b} h^{2}$ & $\mathbf{M}(\mathbf{e V})$ & $Y_{H e}$ & $\ln \left[b^{2} P\left(k_{0}\right)\right]$ & $X$ \\
& & & & & & & & & \\
& & & & & & & & \\
\hline parameters & 0.005 & 0.007 & 0.005 & 0.01 & 0.001 & 0.0002 & $\mathbf{0 . 1 1}$ & 0.01 & 0.007 \\
$+X=N_{\nu}^{\mathrm{r}}$ & 0.005 & 0.008 & 0.005 & 0.01 & 0.003 & 0.0002 & $\mathbf{0 . 1 2}$ & 0.01 & 0.007 \\
$+X=\Omega_{\mathrm{k}}$ & 0.005 & 0.008 & 0.005 & 0.01 & 0.002 & 0.0002 & $\mathbf{0 . 1 3}$ & 0.01 & 0.007 & 0.003 \\
$+X=w$ & 0.005 & 0.008 & 0.005 & 0.01 & 0.002 & 0.0002 & $\mathbf{0 . 1 4}$ & 0.01 & 0.007 & 0.05 \\
$+X=\alpha$ & 0.005 & 0.010 & 0.005 & 0.01 & 0.001 & 0.0002 & $\mathbf{0 . 1 1}$ & 0.02 & 0.007 & 0.008 \\
\hline \hline
\end{tabular}

TABLE II: Absolute errors at the $1-\sigma$ level for various cosmological models, using PLANCK $+\operatorname{SDSS}\left(k_{\max }=0.15 h \mathrm{Mpc}^{-1}\right)$. The first line shows our simplest flat $\Lambda$ CDM model, described by 9 free parameters with fiducial values $C_{200}^{T}=0.85, n_{s}=0.96$, $\tau=0.11, \Omega_{\Lambda}=0.70, \Omega_{m} h^{2}=0.143, \Omega_{b} h^{2}=0.023, M=0.3 \mathrm{eV}$ (normal hierarchy), $Y_{H e}=0.24$. The value chosen for $b^{2} P\left(k_{0}=0.1 \mathrm{~h} \mathrm{Mpc}^{-1}\right)$ is irrelevant. The next lines have one additional parameter $X$ : an effective number of neutrinos $N_{\nu}^{\mathrm{r}}$ parametrizing the abundance of extra relativistic relics, with fiducial value 0 ; a free spatial curvature parametrized by $\Omega_{\mathrm{k}}$ with fiducial value 0 ; a free time-independent equation of state for dark energy parametrized by $w$ with fiducial value -1 ; a free scalar tilt running parametrized by $\alpha=d n_{s} / d \ln k$ with fiducial value 0 .

$M$ are of course bounded from below: the minimal value corresponds to the limit in which the lightest neutrino mass goes to zero, in one of the two NH or IH schemes.

Let us first concentrate on the case in which $\Delta m_{\text {atm }}^{2}$ has its current preferred value of $2.6 \times 10^{-3} \mathrm{eV}^{2}$ (left plot). The minimal value of $M$ in the $\mathrm{NH}$ (resp. $\mathrm{IH}$ ) case is approximately $0.06 \mathrm{eV}$ (resp. $0.10 \mathrm{eV}$ ). However, the $2 \sigma$ detection threshold, defined by $M=2 \sigma(M)$, is around $0.21 \mathrm{eV}$. We conclude that PLANCK+SDSS will probe mainly the region were the three neutrinos are quasi-degenerate in mass, with no possibility to distinguish between the two cases. In absence of clear detection, the $2 \sigma$ upper bound will be of order $0.2 \mathrm{eV}$, corresponding to individual masses $(0.08,0.06$, $0.06) \mathrm{eV}$ assuming $\mathrm{NH}$, or $(0.073,0.073,0.053) \mathrm{eV}$ assuming IH. As expected, we find that the $2 \sigma$ detection threshold is still $0.21 \mathrm{eV}$ when the calculations are performed with a larger value $\Delta m_{\mathrm{atm}}^{2}=3.7 \times 10^{-3} \mathrm{eV}^{2}$ (the $3 \sigma$ upper bound in Eq. 10, as shown in the right plot of Fig. 囵

It is interesting to study whether this precision is limited mainly by a degeneracy between $M$ and some combination of other cosmological parameters, or simply by the experimental sensitivity to the individual effect of $M$. In the first case, the results could be improved by including priors from other types of experiments on the cosmological parameters; in the second case, one would have to wait for a new generation of CMB and/or LSS experiments. In order to address this point, we computed the eigenvectors and eigenvalues of the reduced Fisher matrix. It turns out that for all our fiducial models, one of the unit eigenvectors points precisely in the direction of $M$, with coefficient very close to one in this direction (and, of course, the corresponding eigenvalue matches the error previously obtained for $M$ ). We conclude that $M$ is not affected by a parameter degeneracy, and that independent measurements of other cosmological parameters would not help very much in constraining neutrino masses. Note that this is not yet the case for current cosmological bounds on neutrino masses, where the addition of priors on parameters such as the Hubble constant or $\Omega_{\Lambda}$ leads to more stringent bounds (see e.g. [12]).

The absence of large parameter degeneracies applies to our reference model with nine free parameters. It may not necessarily be true in the presence of extra parameters describing deviations from the concordance $\Lambda$ CDM model. In order to illustrate this point and to test the robustness of our conclusions, we have calculated the error on each parameter for several extended cosmological scenarios, with extra relativistic degrees of freedom, spatial curvature, dark energy with varying density but constant equation of state, or a primordial spectrum with running tilt (see Table III). The neutrino mass bound is found to be quite robust in all these cases, which proves that in none of these models the effect of $M$ can be mimicked by some other parameter combination.

It is also interesting to study the relative impact of CMB temperature, CMB polarization and LSS data on the measurement of $M$. We show in table IIII the error on each parameter for SDSS alone, PLANCK alone (with or without polarization), and various combinations of CMB and LSS data, with an explicit dependence on the value of $k_{\max }$. The complementarity of PLANCK and SDSS clearly appears. While PLANCK alone would achieve only a $1 \sigma$ detection of $M=0.3 \mathrm{eV}$ and SDSS alone would not detect it at all, the combined data would probe this value at the $3 \sigma$ level. One can check from Table [II] that PLANCK data on polarization lowers the error on $M$ by approximately $30 \%$. By diagonalizing the "PLANCK (no pol.)+SDSS" Fisher matrix, we checked that without polarization there would be a significant degeneracy between neutrino mass and optical depth to reionization. Indeed, while reionization lowers the CMB temperature spectrum keeping the matter power spectrum unchanged, the effect of neutrino freestreaming is opposite in first approximation (at least on small scales). So, polarization measurements are indirectly a key ingredient for neutrino mass determination. 


\begin{tabular}{|c|c|c|c|c|c|c|c|c|c|c|}
\hline & $k_{\max }(h / \mathrm{Mpc})$ & $\ln C_{200}^{T}$ & $n_{s}$ & $\tau$ & $\Omega_{\Lambda}$ & $\Omega_{m} h^{2}$ & $\Omega_{b} h^{2}$ & $\mathrm{M}(\mathrm{eV})$ & $Y_{H e}$ & $\ln \left[b^{2} P\left(k_{0}\right)\right]$ \\
\hline \multirow[t]{2}{*}{ SDSS alone } & 0.10 & $\overline{-}$ & 0.6 & $\overline{-}$ & 0.8 & 0.5 & 0.1 & 7.0 & - & 0.3 \\
\hline & 0.15 & - & 0.5 & - & 0.09 & 0.4 & 0.08 & 1.5 & - & 0.06 \\
\hline PLANCK (no pol.) & - & 0.005 & 0.02 & 0.10 & 0.05 & 0.006 & 0.0006 & 0.42 & 0.03 & - \\
\hline \multirow[t]{2}{*}{ PLANCK (no pol.) + SDSS } & 0.10 & 0.005 & 0.02 & 0.08 & 0.02 & 0.002 & 0.0004 & 0.24 & 0.02 & 0.015 \\
\hline & 0.15 & 0.005 & 0.02 & 0.08 & 0.01 & 0.001 & 0.0003 & 0.15 & 0.02 & 0.008 \\
\hline \multirow[t]{3}{*}{ PLANCK (all) + SDSS } & 0.10 & 0.005 & 0.007 & 0.005 & 0.02 & 0.002 & 0.0002 & 0.19 & 0.01 & 0.012 \\
\hline & 0.15 & 0.005 & 0.007 & 0.005 & 0.01 & 0.001 & 0.0002 & 0.11 & 0.01 & 0.007 \\
\hline & 0.20 & 0.004 & 0.007 & 0.005 & 0.006 & 0.0008 & 0.0002 & 0.08 & 0.01 & 0.005 \\
\hline CMBpol & - & 0.003 & 0.003 & 0.003 & 0.006 & 0.0006 & 0.00008 & 0.07 & 0.004 & - \\
\hline CMBpol + SDSS & 0.10 & 0.003 & 0.003 & 0.003 & 0.006 & 0.0006 & 0.00008 & 0.07 & 0.004 & 0.011 \\
\hline
\end{tabular}

TABLE III: Absolute errors at the 1- $\sigma$ level, for various experiments and the same $\Lambda$ CDM model as in table III (with 9 free parameters). In particular, the fiducial value of the total neutrino mass is still $M=0.3 \mathrm{eV}$. When using SDSS, we show the results for three choices of $k_{\max }$, the maximal wavenumber on which the data are compared with linear theory predictions: $k_{\max }=0.10 h \mathrm{Mpc}^{-1}$ (conservative), $0.15 h \mathrm{Mpc}^{-1}$ (reasonable), or $0.20 h \mathrm{Mpc}^{-1}$ (optimistic).

\begin{tabular}{lccc}
\hline \hline & SDSS & SDSS+KAOS & "hypothetical LSS" \\
\hline PLANCK & & & 0.11 \\
CMBpol & 0.21 & 0.16 & 0.09 \\
"ideal CMB" & 0.13 & 0.10 & 0.08 \\
\hline \hline
\end{tabular}

TABLE IV: 2- $\sigma$ detection threshold (in eV) for various combinations of CMB and LSS experiments (assuming the normal hierarchy scenario). The "ideal CMB" experiment is limited only by cosmic variance up to multipole $l=2500$ and covers $100 \%$ of the sky. The "hypothetical LSS" survey has a volume $V_{\text {eff }} \simeq 40(\mathrm{Gpc} / h)^{3}$ and probes the linear spectrum up to $k_{\max }=0.15 h$ $\mathrm{Mpc}^{-1}$ (that would be the case of a large galaxy survey covering $75 \%$ of the sky up to $z=0.8$ ).

\section{B. Post-PLANCK experiments}

Here we consider whether future CMB and LSS experiments will reach a better sensitivity on the neutrino mass, in particular at the level of the small values of $M$ expected for the hierarchical normal and inverted schemes. Sensitivities significantly better than $0.1 \mathrm{eV}$ would mean approaching the absolute minimum of $M$ in the $\mathrm{NH}$ case or even ruling out the IH scenario.

In the previous section, we mentioned a few CMB missions that have been proposed so far in complement to PLANCK. We will study the impact of a few of them, and of an "ideal CMB experiment" that would be limited only by cosmic variance up to $l=2500$ (both for temperature and polarization). The main difficulty for reaching this goal would be to subtract accurately small-scale foregrounds, and in particular point-like sources, but even with current technology such an ideal experiment is conceivable. On the other hand, it is difficult to specify the characteristics of an ideal LSS experiment, since it will be limited by technological improvements in instrumentation and data processing. Therefore, we will keep in the analysis a free parameter $V_{\text {eff }}$ describing the effective volume of an ideal volume-limited survey.

We show in Fig. [6 the predicted $2 \sigma$ error in four cases corresponding to SPTpol (upper left), PLANCK (upper right), CMBpol (lower left), and our ideal CMB experiment (lower right). The value of $2 \sigma$ (in eV) is shown with grey levels, as a function of $k_{\max }$ (horizontal axis) and $V_{\text {eff }}$ (vertical axis) in units of $V_{\text {eff }}$ (SDSS) $=1(\mathrm{Gpc} / h)^{3}$. The total mass has been fixed to $M=0.11 \mathrm{eV}$, distributed according to the $\mathrm{NH}$ scheme. We learned from the previous subsection that for higher values of $M$, the error could be smaller (at most by a factor 2). However, we are now interested in the range $0.05 \mathrm{eV}<M<0.2 \mathrm{eV}$, since larger values should be detected by PLANCK+SDSS, and smaller values are excluded by oscillation experiments. In this range, on can safely interpolate the results obtained at $M=0.11 \mathrm{eV}$. In particular, our results for a cosmic-variance limited CMB experiment are in reasonable agreement with those of [15]. 


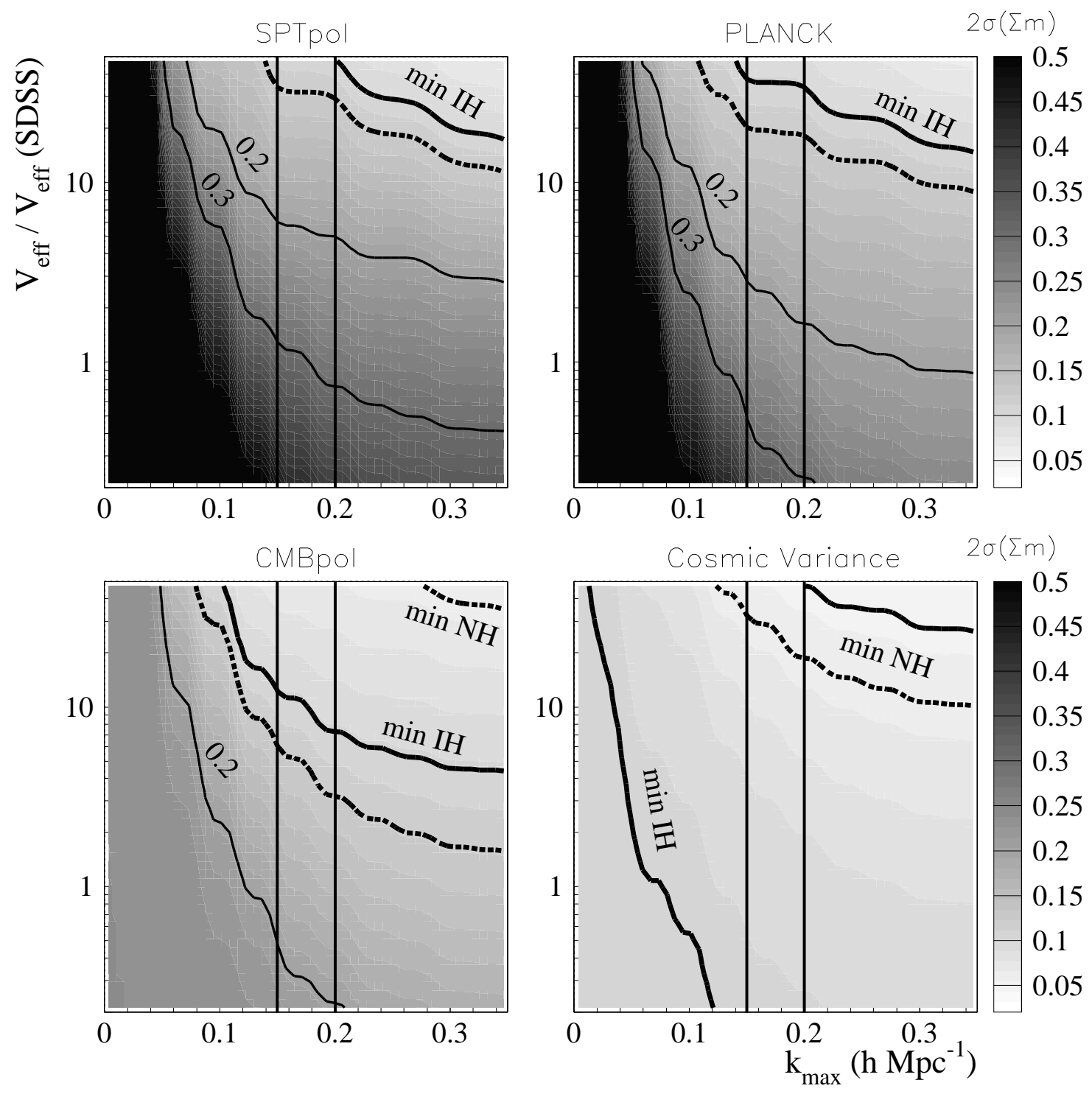

FIG. 6: The grey regions are the $2 \sigma$ expected errors on $\sum m_{\nu}(\mathrm{eV})$ for a fiducial value of $0.11 \mathrm{eV}$, as a function of the parameters of the galaxy survey, where each panel corresponds to a specific CMB experiment. The vertical lines indicate the cut-off wavenumber $k_{\max }$ for the linear matter power spectrum at the conservative (optimistic) value $0.15(0.2) h \mathrm{Mpc}^{-1}$. The thin contours shown are (from bottom to top) for 0.3 and $0.2 \mathrm{eV}$, while the thick contours correspond to the minimum values of $\sum m_{\nu}$ in the IH (lower lines) and NH (upper lines) schemes, assuming the best-fit (solid) or the $3 \sigma$ upper bound (dashed) value of $\Delta m_{\mathrm{atm}}^{2}$.

For SDSS (or for any survey with $z<1$ ) we expect the relevant value of $k_{\max }$ to be around $0.15 h \mathrm{Mpc}^{-1}$. However, depending on the overall amplitude of the matter power spectrum (often parametrized by $\sigma_{8}$, and still poorly constrained) and on future improvements in our understanding of non-linear corrections, this value might appear to be either too optimistic or too pessimistic: this is the reason why it is interesting to leave it as a free parameter.

One can see that replacing PLANCK by CMBpol would lead to a better sensitivity to the neutrino mass, with a $2 \sigma$ detection threshold at $0.13 \mathrm{eV}$ instead of $0.21 \mathrm{eV}$. The expected errors for CMBpol, with and without SDSS data, can be found in Table III] Adding to SDSS the two KAOS surveys (centered around $z=1$ and $z=3$ ) would also lead to some improvement. For Planck+SDSS+KAOS we get a $2 \sigma$ detection threshold of $M \sim 0.16 \mathrm{eV}$, while for CMBpol+SDSS+KAOS one could reach $M \sim 0.10 \mathrm{eV}$. These results are summarized in Table IV

There is still room for improvement beyond this set of experiments. In order to make a precise statement on the conclusions that could be drawn on the long term, we keep the "ideal CMB experiment" characteristics and fix $V_{\text {eff }}$ 

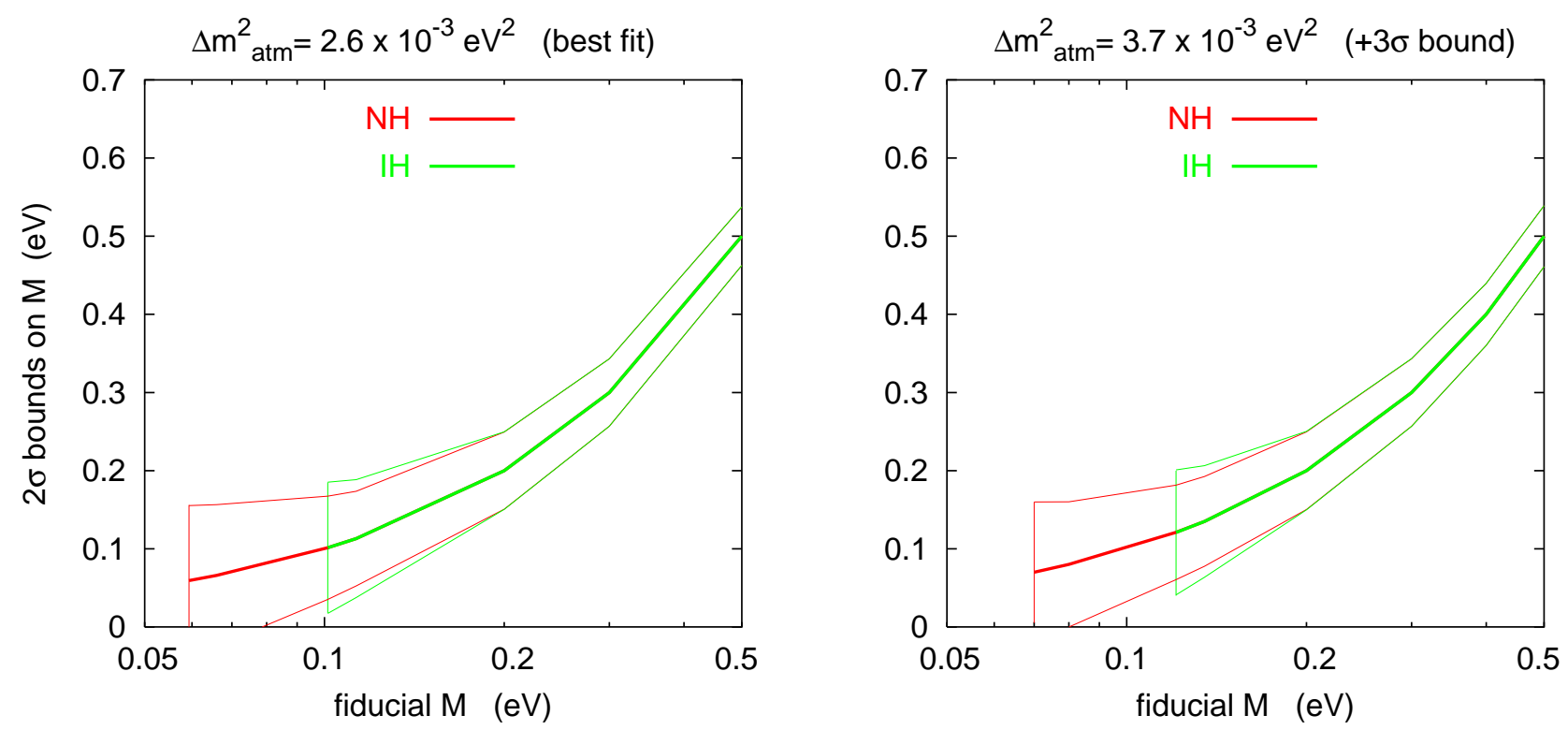

FIG. 7: Predicted $2 \sigma$ error on $\sum m_{\nu}$ as a function of $\sum m_{\nu}$ in the fiducial model, using an ideal CMB experiment (limited only by cosmic variance up to $l=2500$, both for temperature and polarization) and a redshift survey covering $75 \%$ of the sky up to $z \simeq 0.8\left(V_{\text {eff }}=40(\mathrm{Gpc} / h)^{3}\right)$, still limited to $k_{\max }=0.15 h \mathrm{Mpc}^{-1}$. The left plot was obtained with the preferred experimental value of $\Delta m_{\mathrm{atm}}^{2}$, and the right plot with the current $3 \sigma$ upper bound. In each case, we show the results assuming either $\mathrm{NH}$ or IH.

to $40(\mathrm{Gpc} / h)^{3}$ (in section 111 we argued that this could hopefully represent the volume of a survey comparable to the LSST project), while keeping $k_{\max }=0.15 h \mathrm{Mpc}^{-1}$. In Fig. 7 we plot the corresponding results in the same way as we did for PLANCK+SDSS. Assuming the IH scenario, we see that any value of the mass could be detected at the $2 \sigma$ level. Assuming NH, this is only true at the 1 or $1.5 \sigma$ level, depending on the value of $\Delta m_{\text {atm }}^{2}$. The $2 \sigma$ detection threshold is at $0.08 \mathrm{eV}$.

Our results show, for the first time, that if the available cosmological data are precise enough, the expected errors on the neutrino masses depend not only on the sum of neutrino masses, but also on what is assumed for the mass splitting between the neutrino states. As can be seen from Figs. 15 and $\mathbf{7}$ the sensitivity on $M$ will be slightly better in the $\mathrm{NH}$ case in the mass region close to the minimum value of the $\mathrm{IH}$ scheme. These small differences arise from the changes in the free-streaming effect that we have described in section [II and obviously disappear for a total mass in the quasi-degenerate region (above $0.2 \mathrm{eV}$ or so).

In any case, the main contribution of cosmology to the possible discrimination between the neutrino mass schemes will still be the possibility of ruling out the case in which the masses are quasi-degenerate. Even in our most optimistic forecast (Fig. (7), if the preferred value of $M$ turns out to be smaller than $0.1 \mathrm{eV}$, the error bar will still be too large in order to safely rule out the IH case. We also performed an extended analysis in which, instead of assuming either normal or inverted hierarchy, we introduced a tenth free parameter accounting for a continuous interpolation of the mass spectrum between the two scenarios, for fixed $M$. By computing the error on this parameter, we obtained a confirmation that the $\mathrm{NH}$ and $\mathrm{IH}$ scenarios cannot be discriminated directly from the data. However, any analysis of future, very precise cosmological data must take into account the texture of neutrino masses in order to translate the corresponding positive signal (or bound) into $M$.

\section{CONCLUSIONS}

In this paper we have analyzed the sensitivities of future CMB and LSS data to the absolute scale of neutrino masses, taking into account realistic experimental sensitivities and extending the results of previous works [5, 13, 14, 15].

We have considered the values of neutrino masses distributed according to the presently favored three neutrino mass schemes, that follow either a normal or an inverted hierarchy. As discussed in section [II a different distribution of the same total neutrino mass leads to small changes in the cosmological evolution of neutrinos, and in particular in the free-streaming scales (qualitatively discussed, for instance, in [5, 15]). These changes disappear when the total neutrino mass enters the quasi-degenerate region.

We used the Fisher matrix method to forecast the errors on cosmological parameters that can be extracted from 
future CMB experiment and redshift survey data, assuming a fiducial 9-dimensional cosmological model close to the currently favored $\Lambda$ CDM model. Our theoretical CMB and matter power spectra were generated with the standard Boltzmann code CMBFAST, modified in order to include three neutrino states with different masses.

In particular, for the case of PLANCK and SDSS we found good agreement with the results of [13], with a $2 \sigma$-error on the total neutrino mass of $0.2 \mathrm{eV}$ that will allow us to probe only the quasi-degenerate neutrino mass region. Better sensitivity will be achieved with the combination CMBpol and SDSS, for which we found $0.12 \mathrm{eV}$, close to the minimum value of the total neutrino mass in the inverted hierarchy case. These results correspond to a conservative value of $k_{\max }=0.15 \mathrm{~h} \mathrm{Mpc}^{-1}$, the maximal wavenumber on which the LSS data are compared with the predictions of linear theory. We also tested how the errors change when including additional cosmological parameters to our fiducial model. In general, we found that the errors on the neutrino masses are not modified in a significant way.

Our results show that the approach where CMB experiments are only limited by cosmic variance (as in [15]) is probably too simplistic. However, if a future CMB experiment is capable of getting close to such an ideal limit, then the combination with data from galaxy redshift surveys larger than SDSS would lead to errors on the total neutrino mass comparable to the minimum values of the hierarchical scenarios. In such a case, we have shown that there exist slight differences in the expected errors between the two hierarchical neutrino schemes for the same total neutrino mass.

In conclusion, we consider that cosmological data can provide valuable information on the absolute scale of neutrino masses, that nicely complements the present and future projects of beta decay and neutrinoless double beta decay experiments. This conclusion is reinforced when one takes into account other cosmological probes of neutrino masses, complementary to the approach of the present paper. We can cite, for instance, studies of the distribution of matter in the Universe through the distortions of CMB maps caused by gravitational lensing (measured from non-gaussianities in the CMB maps) [17] and the weak gravitational lensing of background galaxies by intervening matter [16, 49, 50, 51].

It is interesting to note that any information on the absolute neutrino masses from cosmology will be interesting not only for theoretical neutrino models, but also for connected baryogenesis scenarios which occur through a leptogenesis process (see e.g. [52, 53, 54]).

\section{Acknowledgments}

We thank Ilenia Picardi for initial discussions concerning the present work, as well as Martin Hirsch and Simon Prunet for various suggestions, and Bruce Bassett for pointing us to the KAOS proposal. This research was supported by a CICYT-IN2P3 agreement. SP was supported by the Spanish grant BFM2002-00345, the ESF network Neutrino Astrophysics and a Ramón y Cajal contract of MCyT.

[1] J. Bonn et al., Nucl. Phys. Proc. Suppl. 91, 273 (2001).

[2] A. Osipowicz et al. [KATRIN Coll.], hep-ex/0109033

[3] S.R. Elliott and P. Vogel, Ann. Rev. Nucl. Part. Sci. 52 115, (2002) hep-ph/0202264.

[4] A.D. Dolgov, Phys. Rept. 370, 333 (2002) hep-ph/0202122.

[5] W. Hu, D.J. Eisenstein and M. Tegmark, Phys. Rev. Lett. 80, 5255 (1998) astro-ph/9712057.

[6] D.N. Spergel et al., Astrophys. J. Suppl. 148, 175 (2003) astro-ph/0302209.

[7] S. Hannestad, JCAP 0305, 004 (2003) astro-ph/0303076.

[8] Ø. Elgarøy and O. Lahav, JCAP 0304, 004 (2003) astro-ph/0303089.

[9] M. Tegmark et al. [SDSS Coll.], Phys. Rev. D, in press astro-ph/0310723.

[10] V. Barger, D. Marfatia and A. Tregre, hep-ph/0312065

[11] S. Hannestad and G. Raffelt, hep-ph/0312154

[12] P. Crotty, J. Lesgourgues and S. Pastor, hep-ph/0402049

[13] D.J. Eisenstein, W. Hu and M. Tegmark, Astrophys. J. 518, 2 (1998) astro-ph/9807130.

[14] J. Lesgourgues, S. Pastor and S. Prunet, Phys. Rev. D 62, 023001 (2000) hep-ph/9912363.

[15] S. Hannestad, Phys. Rev. D 67, 085017 (2003) astro-ph/0211106.

[16] K.N. Abazajian and S. Dodelson, Phys. Rev. Lett. 91, 041301 (2003) astro-ph/0212216 .

[17] M. Kaplinghat, L. Knox and Y.S. Song, Phys. Rev. Lett. 91, 241301 (2003) astro-ph/0303344.

[18] M. Maltoni, T. Schwetz, M.A. Tórtola and J.W.F. Valle, Phys. Rev. D 68, 113010 (2003) hep-ph/0309130.

[19] G.L. Fogli, E. Lisi, A. Marrone and D. Montanino, Phys. Rev. D 67, 093006 (2003) hep-ph/0303064.

[20] M.C. González-García and C. Peña-Garay, Phys. Rev. D 68 (2003) 093003 hep-ph/0306001.

[21] P. Huber, M. Lindner, M. Rolinec, T. Schwetz and W. Winter, hep-ph/0403068

[22] A. Aguilar et al. [LSND Coll.], Phys. Rev. D 64 (2001) 112007 hep-ex/0104049.

[23] M.C. González-García and Y. Nir, Rev. Mod. Phys. 75, 345 (2003) hep-ph/0202058. 
[24] V. Barger, D. Marfatia and K. Whisnant, Int. J. Mod. Phys. E 12, 569 (2003) hep-ph/0308123.

[25] A.D. Dolgov, S.H. Hansen and D.V. Semikoz, Nucl. Phys. B 503, 426 (1997) hep-ph/9703315 .

[26] G. Mangano, G. Miele, S. Pastor and M. Peloso, Phys. Lett. B 534, 8 (2002) astro-ph/0111408.

[27] A.D. Dolgov et al., Nucl. Phys. B 632, 363 (2002) hep-ph/0201287.

[28] A. Lewis and A. Challinor, Phys. Rev. D 66, 023531 (2002) astro-ph/0203507.

[29] J.R. Primack, J. Holtzman, A. Klypin and D.O. Caldwell, Phys. Rev. Lett. 74, 2160 (1995) astro-ph/9411020.

[30] U. Seljak and M. Zaldarriaga, Astrophys. J. 469, 437 (1996) astro-ph/9603033.

[31] J.H. Goldstein et al., Astrophys. J. 599, 773 (2003) astro-ph/0212517.

[32] A.C.S. Readhead et al., astro-ph/0402359

[33] R. Rebolo et al., astro-ph/0402466

[34] M. Bowden et al., astro-ph/0309610

[35] M. Tegmark, D.J. Eisenstein, W. Hu and A. de Oliveira-Costa, Astrophys. J. 530, 133 (2000) astro-ph/9905257.

[36] S. Prunet, S.K. Sethi and F.R. Bouchet, Mon. Not. Roy. Astron. Soc. 314, 358 (2000) astro-ph/9911243.

[37] G. Patanchon, H. Snoussi, J.F. Cardoso and J. Delabrouille, astro-ph/0302078

[38] W. Hu, Phys. Rev. D 65, 023003 (2002) astro-ph/0108090.

[39] W. Hu and T. Okamoto, Astrophys. J. 574, 566 (2002) astro-ph/0111606.

[40] T. Okamoto and W. Hu, Phys. Rev. D 67, 083002 (2003) astro-ph/0301031.

[41] M. Tegmark, Phys. Rev. Lett. 79, 3806 (1997) astro-ph/9706198.

[42] J.A. Tyson [the LSST Coll.], Proc. SPIE Int. Soc. Opt. Eng. 4836, 10 (2002) astro-ph/0302102.

[43] R.E. Lopez, S. Dodelson, A. Heckler and M.S. Turner, Phys. Rev. Lett. 82, 3952 (1999) astro-ph/9803095.

[44] R. Bowen et al., Mon. Not. Roy. Astron. Soc. 334, 760 (2002) astro-ph/0110636].

[45] S. Bashinsky and U. Seljak, Phys. Rev. D, in press astro-ph/0310198.

[46] A. Cuoco, F. Iocco, G. Mangano, G. Miele, O. Pisanti and P.D. Serpico, astro-ph/0307213

[47] R.H. Cyburt, B.D. Fields and K.A. Olive, Phys. Lett. B 567, 227 (2003) astro-ph/0302431.

[48] G. Jungman, M. Kamionkowski, A. Kosowsky and D.N. Spergel, Phys. Rev. Lett. 76, 1007 (1996) astro-ph/9507080.

[49] Y.S. Song and L. Knox, astro-ph/0312175

[50] W. Hu, Astrophys. J. 522, L21 (1999) astro-ph/9904153.

[51] W. Hu, Phys. Rev. D 66, 083515 (2002) astro-ph/0208093.

[52] G.F. Giudice, A. Notari, M. Raidal, A. Riotto and A. Strumia, hep-ph/0310123

[53] T. Hambye, Y. Lin, A. Notari, M. Papucci and A. Strumia, hep-ph/0312203

[54] W. Buchmüller, P. Di Bari and M. Plümacher, hep-ph/0401240 